\title{
Collagen/Hydroxyapatite Bio-Compatible Scaffolds Obtained Through 3D Printing
}

\author{
Madalina Lemnaru, Madalina Georgiana Albu-Kaya², Maria Sonmez ${ }^{2}$, Valentina Mitran ${ }^{3}$, Denisa Ficai ${ }^{1}$, \\ Anton Ficai ${ }^{1}$, Gudovan Dragos ${ }^{1}$, Anisoara Campean ${ }^{3}$, Ecaterina Andronescu ${ }^{1}$ \\ ${ }^{1}$ Department of Science and Engineering of Oxide Materials and Nanomaterials, Faculty of Applied Chemistry and \\ Materials Science, University Politehnica of Bucharest \\ Polizu Street no 1-7, 011061, Bucharest, Romania \\ denisa.ficai@upb.ro \\ ${ }^{2}$ National Research \& Development Institute for Textiles and Leather-division: Leather and Footwear Research Institute, \\ Bucharest, Romania \\ ${ }^{3}$ University of Bucharest \\ Bucharest, Bd. M. Kogalniceanu 36-46 Romania
}

\section{Extended Abstract}

Biocompatible scaffolds are particularly used in medical applications due to reduced systemic toxicity [1,2], but not only, in some life threatening situations such as $3^{\text {rd }}$ or $4^{\text {th }}$ degree burns making the difference between survival and death. The advantage of COLL/HA 3D printed scaffolds come from the porous nature of the resulting material, allowing the adherence of epithelial cells and normal osteoblasts, the resulting material being highly versatile both for tissue engineering and skin regeneration applications.

The printability of hydroxyapatite/collagen mixture depends highly on the ratio between the two components and the printing temperature, and on the $\mathrm{pH}$ of the final printing additive. During the printing process, the vital parameters are the printing speed and the printing temperature. The adherence of the printed material to the surface is also an issue, highly defining the shape of the printed surface. In this paper we obtained a set of optimised printing parameters, printing speed, printing temperature and a composition of the printing mixture, the optimum printing speed being in the $30-50 \mathrm{~mm} / \mathrm{s}$ interval, the optimum temperature between $0-10^{\circ} \mathrm{C}$. In the case of this particular printing mixture, we also found that the rheological properties can be improved by the addition of low viscosity oil content. Due to this aspect, we opted for a volatile oil with antibacterial properties, thus obtaining a dual benefit, that of regenerative purpose material combined with anti-inflamatory or/and antimicrobial benefits. The material's morphology was tested using SEM (scanning electron microscopy), FT-IR microscopy and optical microscopy. The microscopy results confirm the surface homogeneity of the scaffold resulted through 3D printing, the homogeneity of the scaffold being an important requirement for medical applications.

We obtained promising, homogenous 3D printed scaffolds, which can be a highly promising material both for tissue engineering and skin regeneration applications.

Keywords: Collagen, Hydroxyapatite, 3D Printing.

\section{Acknowledgements}

The present work was possible due to the EU-funding grant POSCCE-A2O2.2.1-2013-1, Project No. 638/12.03.2014, code SMIS-CSNR 48652. The financial contribution received from the national project "Structuri poroase biomimetice obtinute prin printare 3D pentru ingineria tesutului osos (Acronim: BIOGRAFTPRINT), Nr 127PED/2017 is also highly acknowledged 


\section{References}

[1] A. Ficai, E. Andronescu, G. Voicu, C. Ghitulica, B. Stefan Vasile, D. Ficai, V. Trandafir, "Self-assembled collagen/hydroxyapatite composite materials," Chemical Engineering Journal, vol. 160, pp. 794-800, 2010.

[2] A. Oryan, S. Alidadi, A. Moshiri, N. Maffulli, "Bone regenerative medicine: classic options, novel strategies, and future directions," Journal of Orthopedic Surgery and Research, vol. 9, no. 1, p.18, 2014. doi: 10.1186/1749-799X9-18. 